\title{
Preliminary design of the wavefront sensor for CCAT
}

David Naylor $^{* a}$, Brad Gom ${ }^{b}$, Mélanie Leclerc ${ }^{c}$, Mathieu Legros ${ }^{c}$, Stephen Padin $^{\mathrm{d}}$, Eugene Serabyn ${ }^{\mathrm{e}}$

${ }^{a}$ Institute for Space Imaging Science, University of Lethbridge, Alberta, T1K 3M4, Canada; ${ }^{b}$ Blue Sky Spectroscopy Inc., 9 - 7404 Avenue South, Lethbridge, Alberta, T1J 0N9, Canada; ${ }^{\mathrm{C}}$ INO, 2740 Einstein St, Québec City, Quebec, G1P 4S4, Canada; ${ }^{\mathrm{d} C a l i f o r n i a}$ Institute of Technology, Pasadena, CA, USA, 91125; ' Jet Propulsion Laboratory, California Institute of Technology, 4800 Oak Grove Drive, Pasadena, CA, USA, 91109

\begin{abstract}
CCAT $^{1}$ is a planned submillimeter telescope currently under development that will be located at an altitude of 5600 meters in the Andes mountains of northern Chile. The atmospheric transmission at this site will allow CCAT to achieve high sensitivity over a wide field of view and a broad wavelength range to provide an unprecedented capability for deep, large area multicolor submillimeter surveys. In order to achieve high aperture efficiencies out to frequencies of $\sim 1 \mathrm{THz}$, the 162 individual panels that form the 25 meter aperture of CCAT must be aligned to a tolerance of a few microns rms. The design of a wavefront sensor to achieve this goal is presented.
\end{abstract}

Keywords: CCAT, submillimeter, telescope, wave front sensor

\section{INTRODUCTION}

$\mathrm{CCAT}^{1}$ is a $25 \mathrm{~m}$ diameter submillimeter telescope currently under development that will operate over the wavelength range from 0.2 to $2 \mathrm{~mm}$. CCAT will be located at an altitude of $5600 \mathrm{~m}$ on Cerro Chajnantor in Northern Chile, close to the ALMA telescope, widely regarded as one of the best submillimeter sites on Earth. The atmospheric transmission at the site provides access to several observing windows as shown in Figure 1.With this broad spectral coverage, wide field of view and suite of sensitive instruments, CCAT will provide astronomers with the capability for deep, large area multicolor submillimeter surveys. In order to achieve high aperture efficiencies up to frequencies of $\sim 1 \mathrm{THz}$, the 162 individual panels that make up the $25 \mathrm{~m}$ aperture of CCAT must be aligned to a tolerance of a few microns, which requires some form of wavefront sensing (WFS). With the emergence of large-format submillimeter wavelength detector arrays $^{3}$, array-based WFS techniques similar to those used at optical/infrared wavelengths could in principle be extended to the submillimeter regime. Their cost and complexity is, however, a serious impediment to their adoption; the control of stray light being a serious concern at these long wavelengths. A number of potential WFS schemes for use at submillimeter wavelengths have been discussed in the literature ${ }^{4,5}$. To date, however, the only proven submillimeter WFS is based on the single pixel shearing interferometer technique ${ }^{6,7}$. While a single-pixel based system is inherently slow and thus subject to systematic errors resulting from atmospheric variations and instrumental drifts during the data acquisition sequence, the technique has been shown to work effectively at the Caltech Submillimeter Observatory (CSO). Moreover, by optimizing the opto-mechanical design, a significant reduction in the time required to map the pupil is expected. For these reasons a WFS based on the shearing interferometer technique has been selected to align the surface of the primary and to position the secondary of CCAT.

\section{WFS REQUIREMENTS}

The top level goals of the CCAT WFS are to measure the wavefront error at both 350 and $850 \mu \mathrm{m}$, with an accuracy better than $4 \mu \mathrm{m} \mathrm{rms}$, in a time of less than $10 \mathrm{~min}$, with more than 50 spatial resolution elements across the pupil diameter, while observing Mars under conditions in which the atmospheric transmission exceeds 0.33 at $350 \mu \mathrm{m}$ and 0.66 at $850 \mu \mathrm{m}\left(2 \mathrm{nd}\right.$ quartile at $\mathrm{EL}=45^{\circ}$ ). Since access to the bent Gregorian focus of CCAT where the WFS will be located is limited, the WFS must be designed so that it can be broken down into sub-components, each weighing $<50 \mathrm{~kg}$, and assembled and aligned in position. The limited access coupled with the complexity of handing liquid cryogens necessitates that the WFS detector be cooled by a closed cycle refrigerator based on pulse tube cooler technology. To

*naylor@uleth.ca ; phone: 1403 329-2426; www.uleth.ca/phy/naylor

Millimeter, Submillimeter, and Far-Infrared Detectors and Instrumentation for Astronomy VII, edited by Wayne S. Holland, Jonas Zmuidzinas, Proc. of SPIE Vol. 9153, 915326 · @ 2014 SPIE · CCC code: $0277-786 \mathrm{X} / 14 / \$ 18 \cdot$ doi: $10.1117 / 12.2055372$ 
minimize sources of potential interference the pulse tube cooler will employ anti-vibration mounts and all electromechanical mechanisms will be suitably shielded and employ brushless motors when practical.

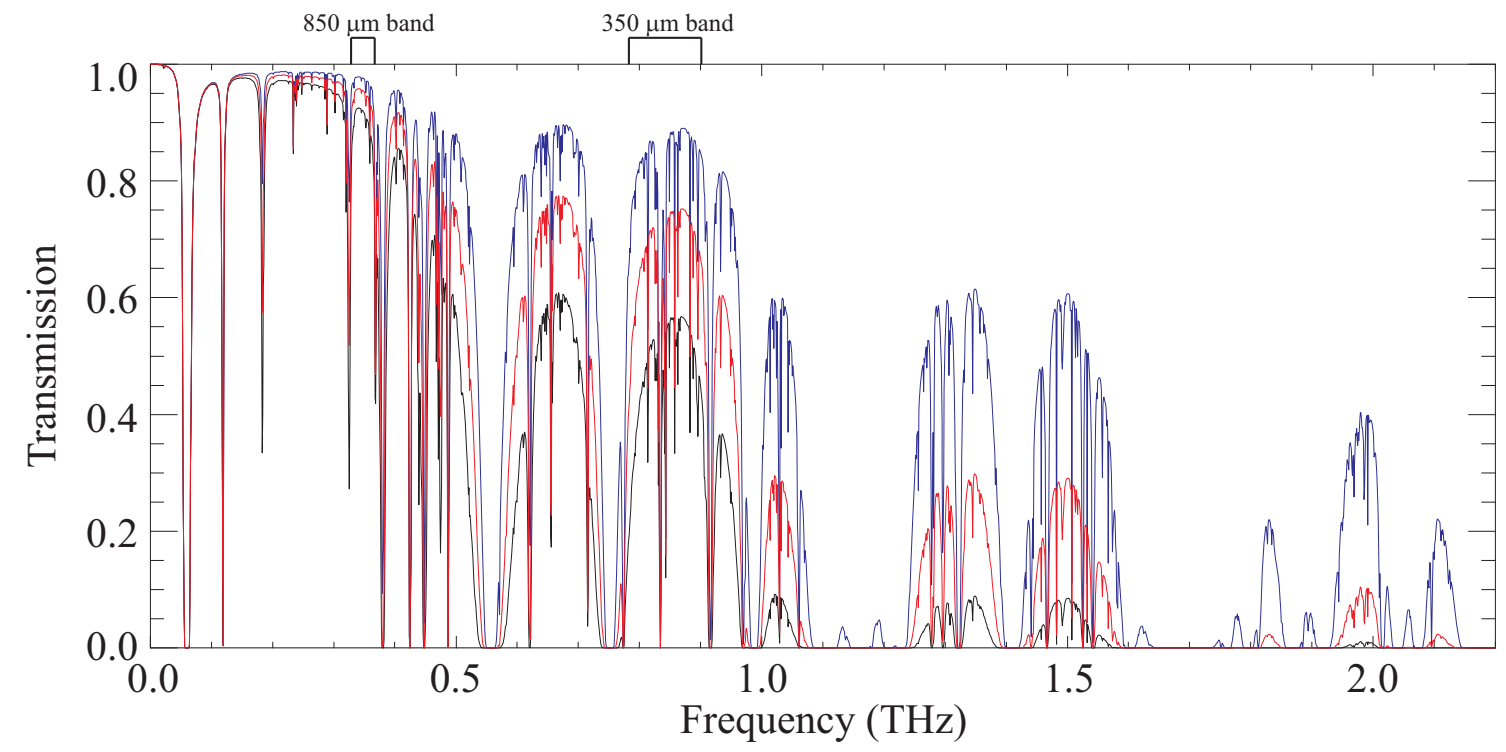

Figure 1. Modelled atmospheric transmission above the CCAT site for water vapour column abundances of $0.1,0.25$ and 0.5 $\mathrm{mm}$, (blue, red, black, respectively) ${ }^{2}$. The 350 and $850 \mu \mathrm{m}$ bands of the WFS are indicated.

\section{WFS TECHNIQUE}

A schematic illustrating the operating principle behind the shearing interferometry technique ${ }^{6}$ is shown in Figure 2 . Two images of the telescope primary mirror are projected onto the two flat mirrors (F1, F2) of a classical Michelson interferometer. The core of the point spread function (PSF) of the telescope from one of the two interferometer mirrors (F1) is used as a reference field on the single pixel detector, and the PSF from the second mirror (F2) is sheared relative to the reference field by a simple rotation of $\mathrm{F} 2$. In this way, $\mathrm{N} \mathrm{x} \mathrm{N}$ off-axis far-field points forming a regular grid are sequentially mapped onto the detector. At each of these positions an interferogram is obtained by scanning the moving mirror of the interferometer (F1). When viewing a point source, the superposition of the on- and off-axis electric fields results in an interferogram that is no longer symmetric. Upon Fourier transformation, the phase information conveyed in the spectrum as a function of both wavelength and off-axis angle can analyzed to produce a phase map of the aperture, which in turn can be expressed as a surface error map ${ }^{6}$.

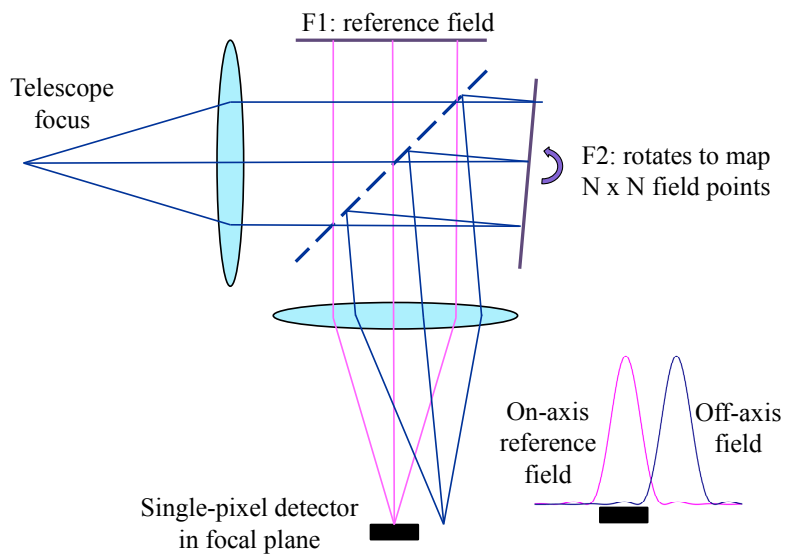

Figure 2. A schematic showing the operating principle of the shearing interferometer ${ }^{6}$. See text for details. 
The shearing interferometer WFS confers several advantages over alternate heterodyne holographic techniques: it is a relatively simple reflective optical design; it is a broadband technique so can employ more sensitive bolometric detectors; it can measure the surface accuracy at two wavelengths with the same optical design. Moreover, observations at the shorter wavelengths suffer less from diffraction effects and are more sensitive to phase variations. Moreover, the shorter wavelengths are those at which the telescope will conduct astronomical imaging observations.

\section{WFS DESIGN}

Figure 3 shows a schematic of the CCAT telescope ${ }^{1}$. The shearing interferometer will be mounted at the bent Gregory focus (shown in the right panel) and is fed by the $\mathrm{f} / 6$ beam from the telescope. Because the WFS moves with the telescope, a key driver was to ensure that the cryogenic detector system will operate over the range of zenith angles for which the WFS will be used. To achieve the required sensitivities, the bolometric detector must be cooled to $\sim 0.3 \mathrm{~K}$, which requires use of a closed cycle ${ }^{3} \mathrm{He}_{-}{ }^{4} \mathrm{He}$ fridge ${ }^{8}$ suspended from the cold plate of the pulse tube cooler. The ${ }^{3} \mathrm{He}-{ }^{4} \mathrm{He}$ fridge normally operates in the vertical position, but can operate out to an angle offset from the vertical of $45^{\circ}$ with acceptably decreased hold time ${ }^{8}$. The pulse tube cooler ${ }^{9}$ itself can operate out to an angle offset from the vertical by $\sim+/-$ $40^{\circ}$. The cycling of both the PTC and ${ }^{3} \mathrm{He}-{ }^{4} \mathrm{He}$ fridges must be done near vertical. In order to allow observations from the zenith to $30^{\circ}$ elevation ( 2 air masses) the cryostat will be mounted at an angle around $40^{\circ}$ with respect to the telescope when the latter is pointing to the zenith.
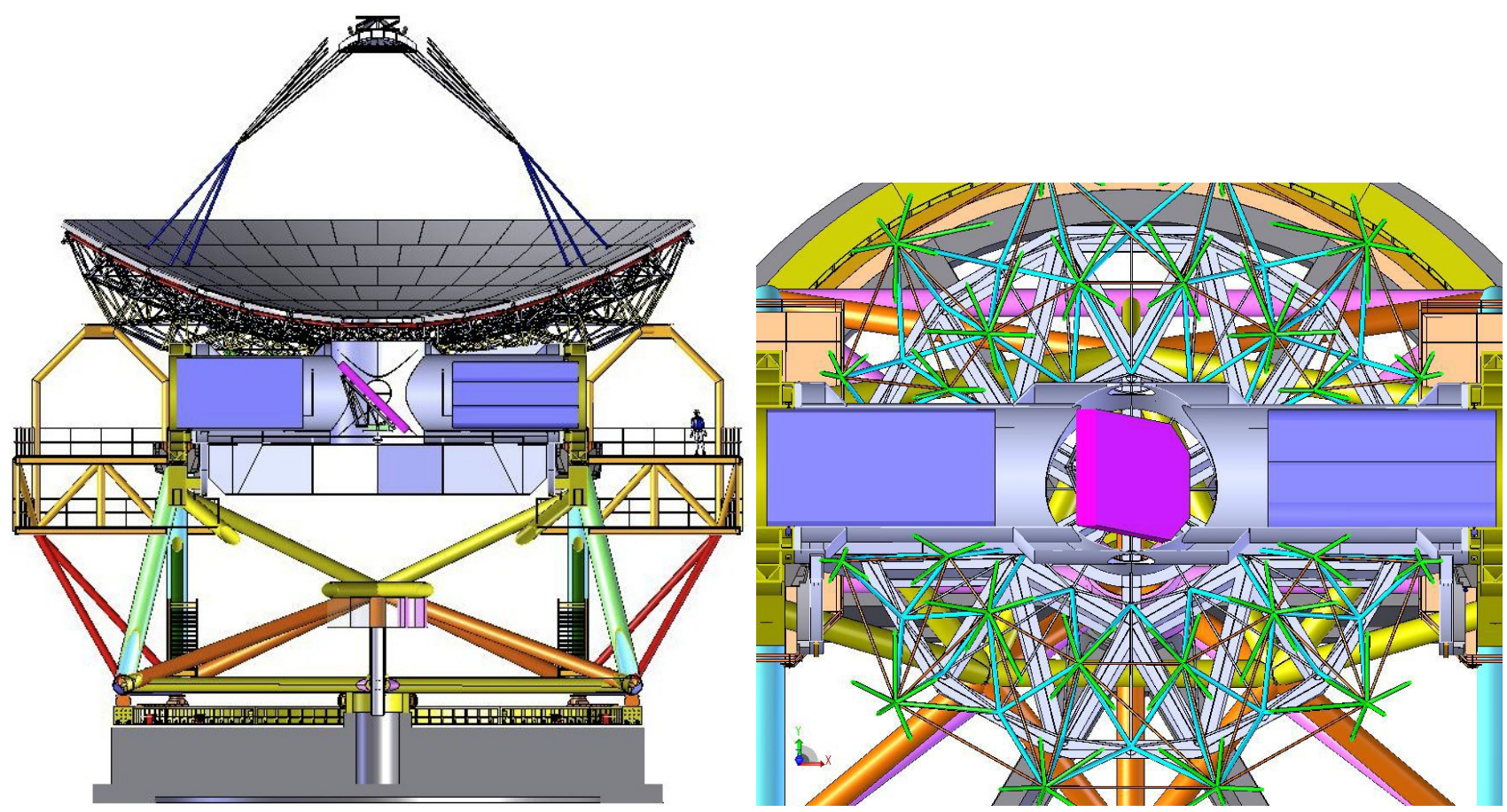

Figure 3: A section of the CCAT telescope through the azimuth and elevation axes (left), and a view of the bent Gregory focus with the primary and upper part of the truss cut away (right). The purple slab is the tertiary mirror.

The space available at the bent Gregorian focus is limited by the complex support structure of the CCAT telescope. It is essential that the WFS does not interfere with the primary mirror truss struts in this area. Figure 4 present two views of the general layout of the components of the WFS with respect to the tertiary mirror (M3). The tilt of the cryostat and the position of the WFS within the allowable envelope can be clearly seen. The mounting flange for the WFS (not shown) will be $1 \mathrm{~m}$ diameter located $2 \mathrm{~m}$ from the center of the tertiary. To give a feel for the scale of the WFS, the volume available is approximately $3 \times 3 \times 3 \mathrm{~m}$, but with restrictions imposed by the truss system. 


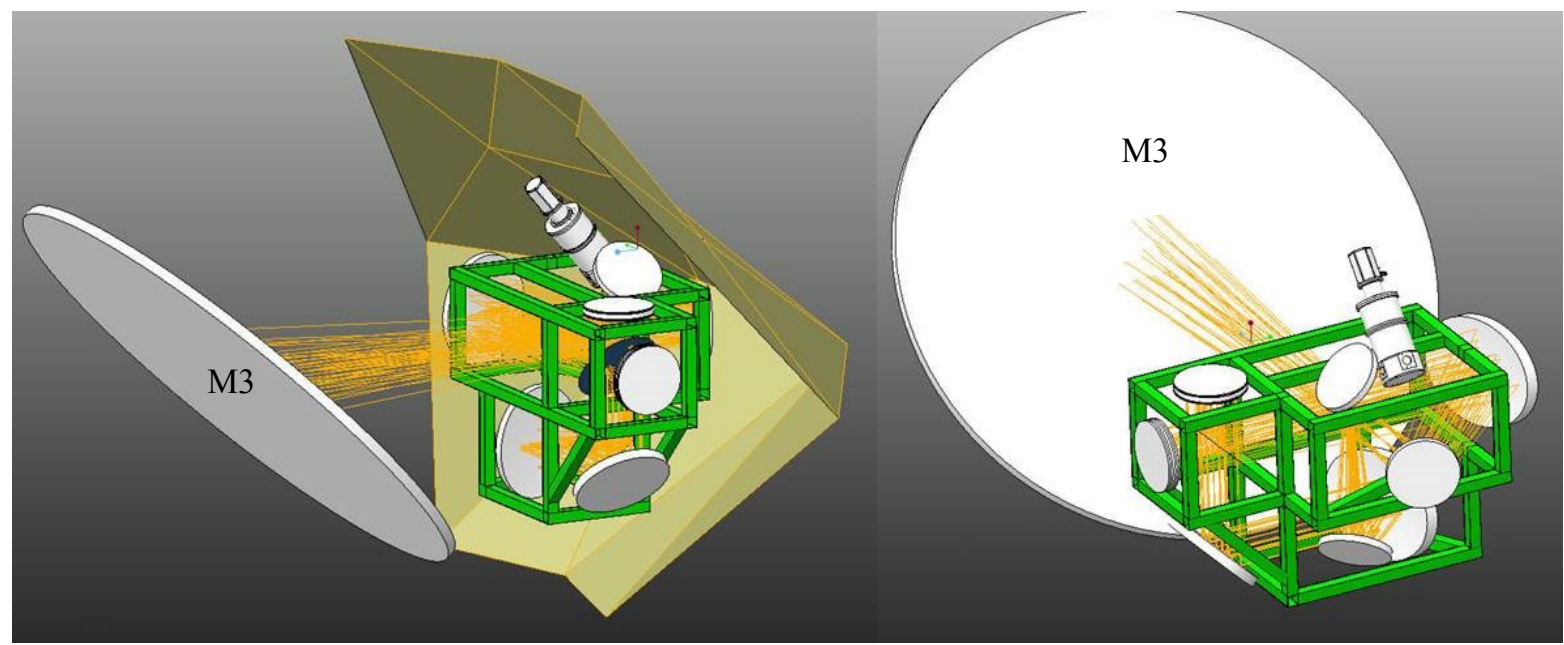

Figure 4. General layout of the WFS as seen looking from behind the tertiary mirror, M3 (left) and towards it (right). The tilted cryostat is seen at the top of the WFS assembly. The green background represents the available envelope.

A second design constraint was that the moving mirror of the interferometer, F1, should always move horizontally to minimize the variation in drive force, and simplify the velocity feedback required for the scanning mirror. A robust and accurate scanning mechanism is necessary to minimize errors in sampling the interferogram, which result in phase errors that would be indistinguishable from phase associated with the shearing interferometer. If the spectral resolving power at $850 \mu \mathrm{m}$ is set equal to the dimension of the far field spatial sampling $(\mathrm{N})$, then for fully double-sided interferograms the total displacement will be a modest $22 \mathrm{~mm}$. Observations at $350 \mu \mathrm{m}$ require less than $10 \mathrm{~mm}$ total displacement. The tilt of the rotating mirror, F2, seen facing downwards in Figure 4 is set by orthogonal electromechanical adjusters with a much lower cadence.

While in its simplest form the WFS consists of two plane and two paraboloidal mirrors, a folded design has been proposed (shown in Figure 5) in order to provide flexibility in the orientation of the cryostat, accommodate the horizontal moving mirror, and to fit within the available space.

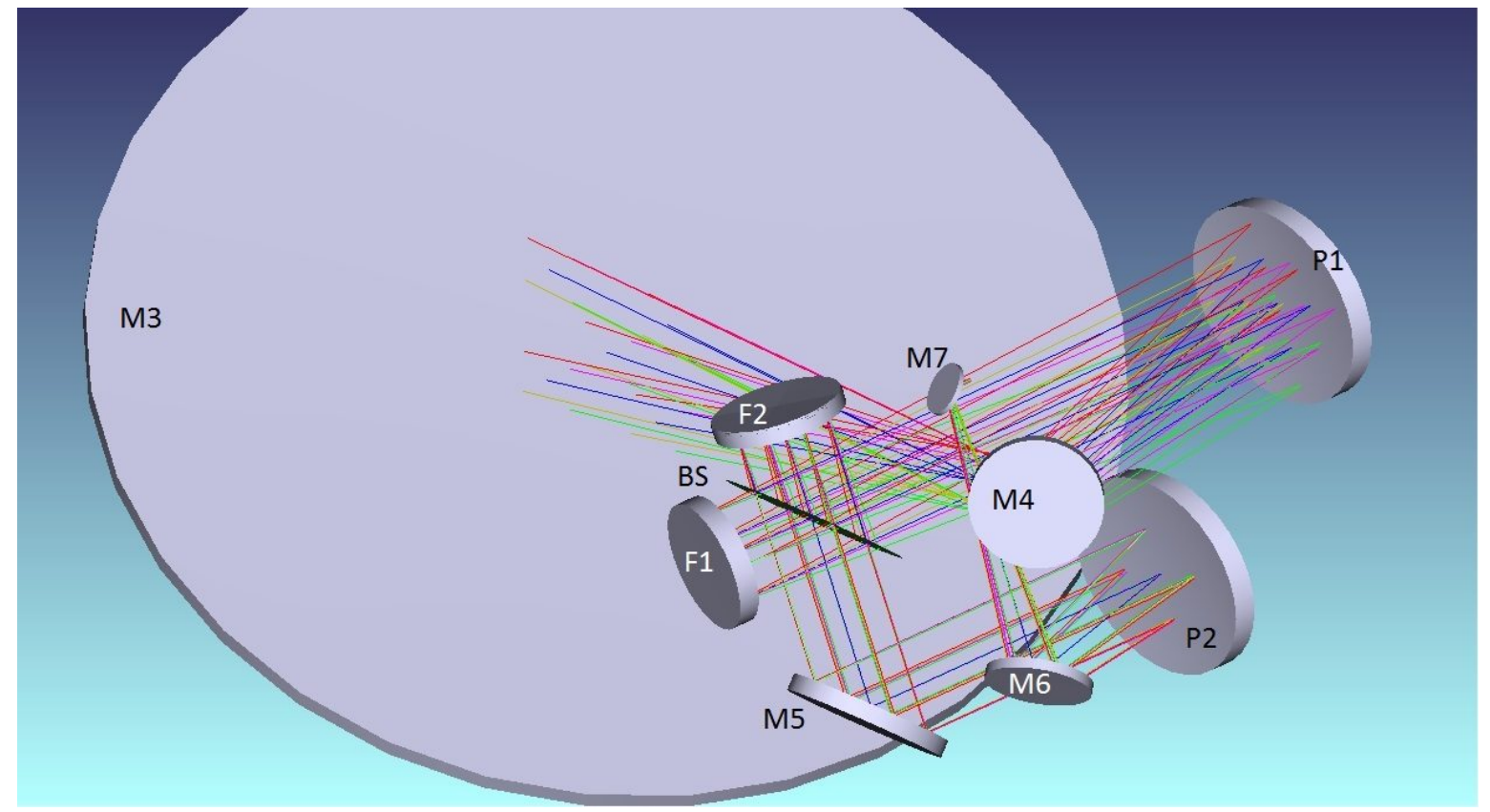

Figure 5. Optical schematic of the WFS showing the tertiary mirror (M3), plane mirrors (M4, M5, M6, M7), plane scanning and tilting mirrors (F1 and F2, respectively), Mylar beamsplitter (BS), and identical paraboloidal mirrors (P1 and P2). 
Light from the tertiary mirror, M3, is directed by a plane folding mirror, M4, to the $840 \mathrm{~mm}$ diameter off-axis paraboloidal mirror, P1. After traversing the Mylar beamsplitter, BS, having an elliptical aperture of $800 \times 500 \mathrm{~mm}$, P1 forms an image of the primary mirror on F1 and F2, each $520 \mathrm{~mm}$ diameter. Two beams from the Michelson interferometer are directed by the folding mirror, M5, to an identical paraboloidal mirror, P2, which is arranged in such a manner (tip to tip) to minimize aberrations ${ }^{10}$. Two further folding mirrors direct the interfering beams into the cryostat, which is tilted so as to maximise the elevation range of operation of the WFS as discussed above, and to fit within the available envelope. The clear aperture of all components is $80 \%$ of their physical size to mitigate diffraction effects.

The initial requirements called for the WFS to operate at wavelengths of 350 and $850 \mu \mathrm{m}$, but not simultaneously. The first solution considered was to have a single detector with a remotely operated cryogenic mechanism to change the band defining filter. However, to operate as a WFS, ideally the beam must be single-moded at each wavelength. A single feedhorn will by necessity be over-moded at shorter wavelengths. Moreover a mechanism to change feedhorns at cryogenic temperatures is impractical. For this reason a dual detector solution, each with its own single mode feedhorn and band defining filter has been selected. The detectors will view the same field though a dichroic similar to those used on the Herschel Space Observatory ${ }^{11,12}$. This solution confers simultaneous operation at both wavelengths and obviates cryogenic mechanisms.

\section{WFS OPTICAL PERFORMANCE}

The optical performance of the WFS configuration shown in Figure 5 has been analyzed using Zemax. At the field center (corresponding to a tilt of zero on F2) the reference and signal paths in the interferometer have small wavefront errors. As the signal path is tilted off-axis, its wavefront error increases because the signal beam moves on the paraboloidal mirror P1. The effect is shown in Figure 6 and results for specific field points are presented in Table 1.

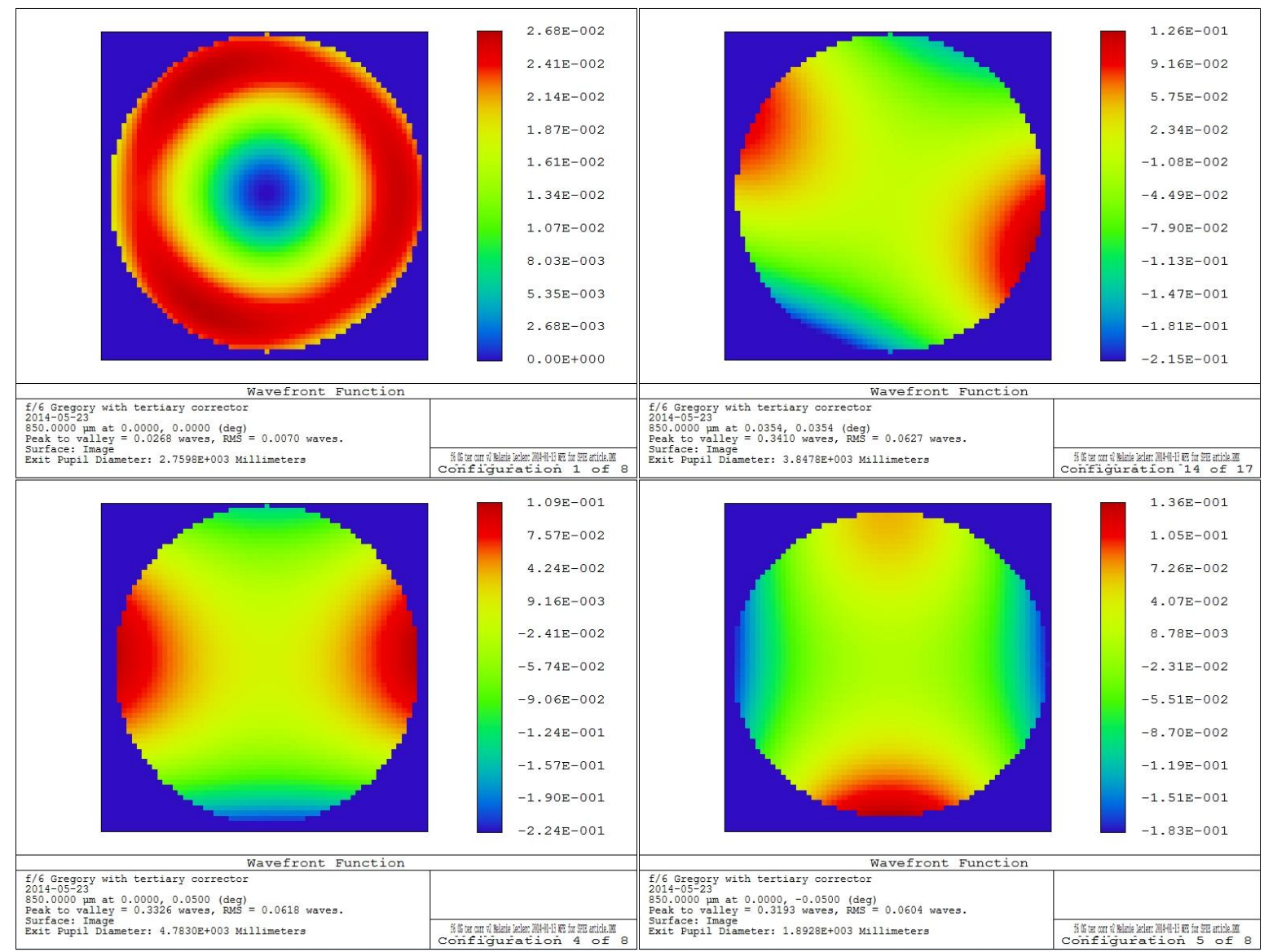

Figure 6. Wavefront error at the field center (top left) and for the field angle extrema $\left(0.0354^{\circ}, 0.0354^{\circ}\right)($ top right $),\left(0.0^{\circ}\right.$, $\left.0.05^{\circ}\right)$ (bottom left) and $\left(0.0^{\circ},-0.05^{\circ}\right)$ (bottom right) for the configuration shown in Figure 5. 
Since CCAT has 6 rings of mirror segments, to measure piston, tip, and tilt, requires two phase measurements across each segment. A $32 \times 32$ point pupil map realizes this objective and requires measurements out to a $0.03^{\circ}$ field radius, corresponding to a tilt of $1.05^{\circ}$ on F2. The WFS design allows for measurements out to a field radius of $0: 05^{\circ}$, which corresponds to a tilt of $1.75^{\circ}$ on F2. As expected, the wave front measurement error (defined as the difference between the wave front error on axis minus the wave front error at field point $(\mathrm{x}, \mathrm{y})$, averaged over the pupil) increases with off axis angle. However, to first order the wave front error increases as the square of the field angle. This can be seen in Table 1 by comparing values for the mean error (last column) for a given field radius (first column). This relationship allows for a simple correction to be applied to the retrieved data based on field radius alone. For the $0.03^{\circ}$ field radius the maximum effect is of order $1 / 200$ of a wave at 850 microns.

Table 1. Wave front measurement error vs. field position for the model of Figure 5.

\begin{tabular}{|c|c|c|c|c|c|c|c|}
\hline \multicolumn{4}{|c|}{ Field position (degrees) } & \multicolumn{2}{|c|}{ F2 tilt (degrees) } & \multicolumn{2}{|c|}{$\langle\mathrm{WFE}(0,0)-\mathrm{WFE}(\mathrm{x}, \mathrm{y})\rangle$} \\
\hline radius & angle & $\mathrm{x}$ & $\mathrm{y}$ & $\mathrm{x}$ & $\mathrm{y}$ & waves at $\lambda=850 \mu \mathrm{m}$ & $\mu \mathrm{m}$ \\
\hline 0.05 & $-\pi$ & 0.05 & 0 & $3.19 \times 10^{-2}$ & $1.73 \times 10^{0}$ & $2.006 \times 10^{-2}$ & 17.05 \\
\hline 0.025 & $-\pi$ & 0.025 & 0 & $7.99 \times 10^{-3}$ & $8.67 \times 10^{-1}$ & $5.004 \times 10^{-3}$ & 4.25 \\
\hline 0.0125 & $-\pi$ & 0.0125 & 0 & $2.00 \times 10^{-3}$ & $4.34 \times 10^{-1}$ & $1.245 \times 10^{-3}$ & 1.06 \\
\hline 0.05 & $\pi$ & -0.05 & 0 & $3.20 \times 10^{-2}$ & $-1.73 \times 10^{0}$ & $2.014 \times 10^{-2}$ & 17.12 \\
\hline 0.025 & $\pi$ & -0.025 & 0 & $8.00 \times 10^{-3}$ & $-8.68 \times 10^{-1}$ & $5.053 \times 10^{-3}$ & 4.29 \\
\hline 0.0125 & $\pi$ & -0.0125 & 0 & $2.00 \times 10^{-3}$ & $-4.34 \times 10^{-1}$ & $1.270 \times 10^{-3}$ & 1.08 \\
\hline 0.05 & $\pi / 2$ & 0 & 0.05 & $-1.70 \times 10^{0}$ & $-2.42 \times 10^{-4}$ & $2.002 \times 10^{-2}$ & 17.02 \\
\hline 0.025 & $\pi / 2$ & 0 & 0.025 & $-8.60 \times 10^{-1}$ & $-6.13 \times 10^{-5}$ & $5.022 \times 10^{-3}$ & 4.27 \\
\hline 0.0125 & $\pi / 2$ & 0 & 0.0125 & $-4.32 \times 10^{-1}$ & $-1.54 \times 10^{-5}$ & $1.257 \times 10^{-3}$ & 1.07 \\
\hline 0.05 & $-\pi / 2$ & 0 & -0.05 & $1.77 \times 10^{0}$ & $-2.41 \times 10^{-4}$ & $2.021 \times 10^{-2}$ & 17.18 \\
\hline 0.025 & $-\pi / 2$ & 0 & -0.025 & $8.75 \times 10^{-1}$ & $-6.08 \times 10^{-5}$ & $5.043 \times 10^{-3}$ & 4.29 \\
\hline 0.0125 & $-\pi / 2$ & 0 & -0.0125 & $4.36 \times 10^{-1}$ & $-1.52 \times 10^{-5}$ & $1.259 \times 10^{-3}$ & 1.07 \\
\hline 0.05 & $\pi / 4$ & 0.03535 & 0.03535 & $-1.20 \times 10^{0}$ & $1.23 \times 10^{0}$ & $1.998 \times 10^{-2}$ & 16.98 \\
\hline
\end{tabular}

\section{WFS SENSITIVITY}

While at first glance it may seem that extending the proven shearing interferometer concept from the $10.4 \mathrm{~m} \mathrm{CSO}$ telescope to the $25 \mathrm{~m}$ CCAT telescope would be straightforward, it quickly becomes evident that the requirement that the WFS sources have bright, broad-band emission and are point like (scaling as $\lambda / D$ ), limits the choice of planets, the only potential astronomical candidates. Indeed, Mars is the optimum target, but is only suitable when it is at the lower end of its range of angular diameter. This should come as no surprise since the relevant metric in the shearing interferometer is the source flux received per measurement subaperture ${ }^{4}$, which is independent of telescope size. It is instructive to study the use of Mars as the WFS source. To first order, the flux density from Mars $\left(\sim 10^{9}\right.$ photons $\left.\mathrm{m}^{-2} \mathrm{~s}^{-1}\right)$ changes little with wavelength across the submillimeter spectral range. A theoretical analysis for a pupil plane $\mathrm{WFS}^{4}$, based on reasonable assumptions concerning the signal and background photon flux measured with an ideal WFS when observing Mars as a point source, shows that the rms surface error, $\sigma(\mu \mathrm{m})$, in a $1 \mathrm{~m}^{2}$ sub-aperture, can be expressed as:

$$
\sigma \approx 1.610^{-3} \lambda \sqrt{\frac{1+n}{t}}
$$

in a measurement time $t$ (seconds) at a wavelength, $\lambda(\mu \mathrm{m})$, where $n$ is the occupancy of the background mode, which in the Rayleigh-Jeans limit is $\sim k T / h v$. Assuming a similar response for the shearing WFS, the rms surface measurement accuracy in one second is 6 and $1.5 \mu \mathrm{m}$ at 850 and $350 \mu \mathrm{m}$, respectively. In practice, an additional factor of 2 in time is required to obtain the "off-source" map, required to subtract the dominant background emission term. Regardless, the time needed to reach a measurement uncertainty of $1 \mu \mathrm{m} \mathrm{rms}$ for a sub-aperture of $1 \mathrm{~m}^{2}$ is $\sim 1$ and $\sim 0.1$ minutes at 850 and $350 \mu \mathrm{m}$, respectively.

These theoretical numbers, however, differ significantly from the practical WFS measurements obtained at the $\mathrm{CSO}^{6}$ which took $\sim 2$ hours to map a grid of $21 \times 21$ far field points, at $850 \mu \mathrm{m}$, and yielded surface errors of $\sim 10 \mu \mathrm{m}$. There are several well understood reasons for this inefficiency most associated with the telescope and instrument overhead ${ }^{6}$. The theoretical results suggest that a significant potential improvement exists if the overheads can be mitigated. 
Overheads aside, one of the remaining unresolved issues is the ability to reach detector NEPs of a few times $10^{-15}$ $\mathrm{W} / \sqrt{\mathrm{Hz}}$ with a cryogen free system that are necessary for the WFS $\mathrm{W}^{6}$. The high impedance of semiconducting Si detectors makes them extremely sensitive to vibrations. To our knowledge, no semiconductor bolometer has been successfully reached these NEPs when operated with a pulse tube cooler. While a wet cryostat detector system provides a proven alternative, as noted above, transferring liquid cryogens (both Nitrogen and Helium) within the limited space available at the bent Gregorian focus makes this solution unattractive. The use of lower impedance detectors which are far less susceptible to noise pick-up is being explored. Several potential options exist, from single pixel superconducting Transition Edge Sensors (TES) to Kinetic Inductance Detectors (KID). A KID single pixel detector is a more favorable option, but requires further development. Fortunately, the field of submillimeter detector development continues to advance at an astonishing rate, with many papers on the subject being presented at this conference. While some development work will likely be required, the prospects for a cryogen free detector solution with the required sensitivity would appear promising.

\section{CONCLUSIONS AND FUTURE WORK}

We have presented the preliminary design of the CCAT WFS based on a shearing interferometer. The design minimizes optical aberrations and uses fold mirrors to accommodate constraints arising from the orientation of the cryogenic detector system, the moving mirror system, and the space envelope within the telescope truss structure. The design uses two detectors, each with a feedhorn and filters optimized for the 850 and $350 \mu \mathrm{m}$ bands. This solution not only eliminates the need for cryogenic mechanisms, but also provides simultaneous observations of the telescope surface at both wavelengths. This will provide an independent measure of the surface accuracy under the same observing conditions, and indeed at the same wavelengths at which the observatory will perform its science observations.

We are currently studying the positioning tolerance analysis for the optical components as input to the mechanical design of the optical mounts and structural sub-modules. The next step will be to perform a structural finite element analysis to estimate the framework deformation under different telescope orientations. The detector remains an open issue. While a wet cryostat provides a backup solution it is far from ideal from a detector NEP perspective. We are currently testing a semiconducting detector in a cryogen free system to investigate the noise issues and explore if they can be mitigated. In parallel we are investigating low impedance detector solutions.

\section{REFERENCES}

[1] http://www.ccatobservatory.org/index.cfm

[2] http://blueskyspectroscopy.com/products-and-services/btram-v3/

[3] Holland, W. S. et al., "SCUBA-2: the 10000 pixel bolometer camera on the James Clerk Maxwell Telescope," Monthly Notices of the Royal Astronomical Society 430, 2513 (2013).

[4] Serabyn, E., "Fundamental limits to wavefront sensing in the submillimeter," Proc. SPIE 62750Z, (2006).

[5] Serabyn, E. and Wallace, J. K., "Submillimeter pupil-plane wavefront sensing," Proc. SPIE 77410U, (2010).

[6] Serabyn, E., Phillps, T. G., and Masson, C. R., "Surface figure measurements of radio telescopes with a shearing interferometer," Applied Optics 30, 1227 (1991).

[7] Woody, D., Serabyn, E., and Schinkel, A., "Measurement, modeling, and adjustment of the 10.4-m-diameter Leighton telescopes," Proc. SPIE 3357, 474 (1998).

[8] http://www.chasecryogenics.com/

[9] http://www.cryomech.com/

[10] James, J. F. and Sternberg, R. S., [Design of Optical Spectrometers], Chapman and Hall, London, (1969).

[11] Pilbratt, G. et al., "Herschel Space Observatory - An ESA facility for far-infrared and submillimetre astronomy," Astronomy and Astrophysics 518, L1 (2010).

[12] Griffin, M. J., et al., "The Herschel-SPIRE instrument and its in-flight performance," Astronomy and Astrophysics, 518, L3 (2010). 